\title{
ON THE SOLUTION OF THE LIOUVILLE EQUATION OVER A RECTANGLE
}

\author{
A.M. ARTHURS, J. CLEGG ${ }^{1}$ and A.K. NAGAR \\ University of York \\ Department of Mathematics \\ Heslington, York Y01 5DD \\ United Kingdom
}

(Received September, 1992; Revised October, 1995)

\begin{abstract}
Methods for integral equations are used to derive pointwise bounds for the solution of a boundary value problem for the nonlinear Liouville partial differential equation over a rectangle. Several test calculations are performed and the resulting solutions are more accurate than those obtained previously by other methods.

Key words: Nonlinear, Liouville, Partial Differential Equation, Boundary Value Problem, Hilbert Space, Eigenvalue, Integral Equation, Green's Function, Hilbert-Schmidt Kernel, Bounded Completely Continuous Integral Operator, Norm of Operator, Contraction Mapping, Pointwise, Bounds, Method of Hooke and Jeeves.
\end{abstract}

AMS (MOS) subject classifications: $35 \mathrm{G} 30,41 \mathrm{~A} 99,45 \mathrm{H} 05,45 \mathrm{P} 05,65 \mathrm{M} 99$.

\section{Introduction}

The boundary value problem involving the two-dimensional Liouville equation

$$
\frac{\partial^{2} \varphi}{\partial x^{2}}+\frac{\partial^{2} \varphi}{\partial y^{2}}=-e^{-\varphi} \quad \text { in } \Re
$$

where $\mathscr{B}$ denotes the rectangle

$$
\mathscr{B}=\{0 \leq x \leq a, 0 \leq y \leq b\}
$$

and with the boundary condition

$$
\varphi=0 \text { on } \partial \mathscr{\Re}
$$

arises in hydrodynamics (cf. [2]) where $\varphi$ is the stream function. Because of its nonlinear character, little is known about the solution of equations (1.1) and (1.3).

Approximate solutions of this problem have been obtained by means of two different approaches. One of these [3] used a quasilinearization technique to arrive at a numerical solution of the particular case $a=\frac{1}{2}, b=\frac{1}{4}$. The other approach [1] employed complementary variational

\footnotetext{
${ }^{1}$ Present address: Department of Electronics, University of York, England. 
principles to obtain a variational solution of the two cases $a=1, b=1$ and $a=\frac{1}{2}, b=\frac{1}{4}$. Neither of these methods provides a pointwise error for their approximate solutions. The quasilinearization technique is an iterative method and is considered to have converged when the two latest iterates differ by less than a preassigned small quantity, whereas in the variational approach, an average error estimate over the whole region $\mathscr{B}$ is provided. In the present paper, we adopt a quite different approach which provides pointwise bounds for the solution. Our method uses an integral equation formulation and some simple bounding results. This approach proves to be relatively straightforward to implement and in the test cases considered leads to close pointwise bounds for the solution.

Our first step is to find out as much as possible about the function $\varphi$ from the equation (1.1) with boundary condition (1.3). Suppose the function $\varphi$ has a local minimum at an interior point $P$ of $\mathscr{B}$. Then from elementary calculus, it follows that

$$
\frac{\partial^{2} \varphi}{\partial x^{2}} \geq 0, \quad \frac{\partial^{2} \varphi}{\partial y^{2}} \geq 0 \quad \text { at } P .
$$

Now this contradicts the fact that from (1.1), the inequality

$$
\frac{\partial^{2} \varphi}{\partial x^{2}}+\frac{\partial^{2} \varphi}{\partial y^{2}}<0
$$

holds at every point in the region $\mathscr{R}$. We can therefore deduce that $\varphi$ cannot attain its minimum at any interior point of $\Re$ and so its minimum value is reached on the boundary $\partial \Re$ of $\Re$. This is an example of a result proved in $[4,7]$. Since $\varphi=0$ on $\partial \mathscr{B}$ we therefore arrive at the following property of the solution function

$$
\varphi(x, y) \geq 0, \quad(x, y) \in \Re+\partial \mathscr{R} .
$$

A simple upper bounding function for the solution $\varphi(x, y)$ can be found as follows. If we write

$$
L=-\frac{\partial^{2}}{\partial x^{2}}-\frac{\partial^{2}}{\partial y^{2}}, \quad(x, y) \in \Re
$$

equation (1.1) takes the form

$$
L \varphi=e^{-\varphi} \text { in } \Re .
$$

Now consider the problem

$$
\begin{aligned}
& L \psi=1 \text { in } \Re \\
& \psi=0 \text { on } \partial \Re .
\end{aligned}
$$

Then we have

$$
\begin{gathered}
L(\psi-\varphi)=1-e^{-\varphi} \geq 0 \text { in } \Re \\
\psi-\varphi=0 \text { on } \partial \Re .
\end{gathered}
$$

Hence, by the maximum principle (cf. [7]),

$$
\psi-\varphi \geq 0 \text { for all }(x, y) \in \mathscr{R}+\partial \mathscr{R}
$$


that is, $\psi(x, y)$ is an upper bounding function for $\varphi(x, y)$. For each rectangle $\mathscr{B}$ we find from (1.7) and (1.8) that the function $\psi$ is given by

$$
\begin{gathered}
\psi(x, y)=L^{-1} .1 \\
=\int_{0}^{b} \int_{0}^{a} k_{0}(x, y, s, t) d s d t .
\end{gathered}
$$

where the kernel $k_{0}$ is the Green's function for (1.7), (1.8) given by expression (2.3) with $\mu_{n}$ replaced by $\frac{n \pi}{b}$.

The boundary value problem in (1.1) to (1.3) can be reformulated as an integral equation

$$
\varphi=L^{-1} e^{-\varphi}
$$

but the method we wish to use does not apply to this formulation because $L^{-1} e^{-\varphi}$ is not a contraction operator for all rectangles $\Re$. To find a suitable contraction we need to reduce the norm of $L^{-1}$ and we do this by introducing the operator

$$
A=L+\sigma^{2} I,
$$

where $\sigma$ is an arbitrary real parameter at this stage and $I$ is the identity operator. We therefore consider the problem

$$
\begin{aligned}
& \text { find } \varphi=\varphi(x, y) \text { : } \\
& \qquad A \varphi \equiv-\frac{\partial^{2} \varphi}{\partial x^{2}}-\frac{\partial^{2} \varphi}{\partial y^{2}}+\sigma^{2} \varphi=\sigma^{2} \varphi+e^{-\varphi} \equiv f(\varphi) \text { in } \mathscr{R}
\end{aligned}
$$

subject to

$$
\varphi=0 \text { on } \partial \mathscr{R} \text {. }
$$

Now we let $\mathfrak{H} 6$ be the Hilbert space of all real-valued functions of two variables defined on $\Re_{0}$ which are square-integrable. The inner product on 36 is given by

$$
\langle u(x, y), v(x, y)\rangle=\int_{0}^{b} \int_{0}^{a} u(x, y) v(x, y) d x d y,
$$

and the norm is defined by

$$
\|u\|=\langle u, u\rangle^{\frac{1}{2}}=\left(\int_{0}^{b} \int_{0}^{a} u(x, y)^{2} d x d y\right)^{\frac{1}{2}} .
$$

The operator $A$ in (1.14) with boundary condition (1.16) is both positive and self-adjoint in the Hilbert space $\mathfrak{H} \mathcal{G}$, and its lowest eigenvalue is

$$
\lambda_{0}=\pi^{2}\left(\frac{1}{a^{2}}+\frac{1}{b^{2}}\right)+\sigma^{2} .
$$




\section{Integral Equation Form}

If we introduce the inverse $K$ of the operator $A$ given in (1.15), subject to the boundary condition (1.16), we find that $\varphi$ satisfies the equivalent integral equation

$$
F \varphi \equiv \varphi-K\{f(\varphi)\}=0
$$

that is,

$$
\varphi(x, y)=K f(\varphi)=\int_{0}^{b} \int_{0}^{a} k(x, y ; s, t) f\{\varphi(s, t)\} d s d t
$$

where $f$ is defined by $(1.15)$ and $k(s, y ; s, t)$ is the kernel of $K$, that is the Green's function (cf. [5])

$$
k(x, y ; s, t)= \begin{cases}\frac{2}{b} \sum_{n=1}^{\infty} \frac{\sinh \mu_{n} x \sinh \mu_{n}(a-s) \sin \frac{n \pi y}{b} \sin \frac{n \pi t}{b}}{\mu_{n} \sinh \mu_{n} a} & x<s, \\ \frac{2}{b} \sum_{n=1}^{\infty} \frac{\sinh \mu_{n} s \sinh \mu_{n}(a-x) \sin \frac{n \pi y}{b} \sin \frac{n \pi t}{b}}{\mu_{n} \sinh \mu_{n} a} & x>s,\end{cases}
$$

where

$$
\mu_{n}^{2}=\frac{n^{2} \pi^{2}}{b^{2}}+\sigma^{2}
$$

By (1.4) and (1.11) we know that we are seeking the solution $\varphi$ of (2.1) which belongs to the subset $\varphi$ of $J 6$ given by

$$
\varphi=\left\{\varphi_{i}(x, y): 0 \leq \varphi_{i} \leq \bar{\varphi}, \quad 0 \leq x \leq a, 0 \leq y \leq b\right\},
$$

where, for example, $\bar{\varphi}=\max \psi(x, y)$ over $\Re$.

Since the kernel $k(x, y ; s, t)$ in $(2.3)$ is symmetric with respect to interchange of $(x, y)$ and $(s, t)$, and is Hilbert-Schmidt, $K$ is a bounded completely continuous operator (cf. [9]). Furthermore, since $K$ is a positive operator, it follows that the norm $\|K\|$ of $K$, defined by

$$
\|K\|=\max \langle K v, v\rangle \text { with }\|v\|=1,
$$

is equal to the largest eigenvalue of $K$, that is

$$
\begin{aligned}
\|K\|= & (\text { lowest eigenvalue of } A)^{-1} \\
& =\left(\frac{\pi^{2}}{a^{2}}+\frac{\pi^{2}}{b^{2}}+\sigma^{2}\right)^{-1}
\end{aligned}
$$

on using (1.19).

We can now establish some useful inequalities involving $f, K f$ and $F$. For any functions $\varphi_{1}$ and $\varphi_{2}$ in the set $\varphi$ of (2.5) we show that there are parameters $\alpha, \beta$ and $\gamma$ such that

$$
\begin{array}{cl}
\left\|f\left(\varphi_{1}\right)-f\left(\varphi_{2}\right)\right\| \leq \alpha\left\|\varphi_{1}-\varphi_{2}\right\|, & \alpha>0 \\
\left\|K f\left(\varphi_{1}\right)-K f\left(\varphi_{2}\right)\right\| \leq \beta\left\|\varphi_{1}-\varphi_{2}\right\|, & 0<\beta<1
\end{array}
$$


and

$$
\left\|F \varphi_{1}-F \varphi_{2}\right\| \geq \gamma\left\|\varphi_{1}-\varphi_{2}\right\|, \quad \gamma=1-\beta>0
$$

For the function $f$ in (1.15), we have by the mean value theorem, that

$$
\begin{gathered}
\left\|f\left(\varphi_{1}\right)-f\left(\varphi_{2}\right)\right\|=\left\|f^{\prime}(\theta)\left(\varphi_{1}-\varphi_{2}\right)\right\|, \quad \theta \in\left[\varphi_{1}, \varphi_{2}\right], \\
=\left\|\left(\sigma^{2}-e^{-\theta}\right)\left(\varphi_{1}-\varphi_{2}\right)\right\| \\
\leq \max _{s, t \in \Re_{R}}\left|\sigma^{2}-e^{-\theta}\right|\left\|\varphi_{1}-\varphi_{2}\right\|,
\end{gathered}
$$

which establishes (2.8) with

$$
\alpha=\max _{s, t \in \mathscr{R}}\left|\sigma^{2}-e^{-\theta(s, t)}\right|
$$

If we write

$$
m=\exp (-\bar{\varphi})
$$

then

$$
0<m \leq e^{-\theta(s, t)}<1, \quad(s, t) \in \mathscr{B}
$$

Now choose the value of $\sigma^{2}$ to minimize the value of $\alpha$ in (2.11), that is, take

$$
\sigma^{2}-m=1-\sigma^{2}
$$

giving

and

$$
\sigma^{2}=\frac{1+m}{2}
$$

$$
\alpha=\frac{1-m}{2}
$$

For the operator $K f$ of (2.2), we have

$$
\begin{gathered}
\left\|K f\left(\varphi_{1}\right)-K f\left(\varphi_{2}\right)\right\| \leq\|K\|\left\|f\left(\varphi_{1}\right)-f\left(\varphi_{2}\right)\right\| \\
\leq \alpha\|K\|\left\|\varphi_{1}-\varphi_{2}\right\|
\end{gathered}
$$

by (2.8). Thus the inequality in (2.9) holds with

$$
\begin{gathered}
\beta=\alpha\|K\| \\
=\alpha\left(\frac{\pi^{2}}{a^{2}}+\frac{\pi^{2}}{b^{2}}+\sigma^{2}\right)^{-1} \\
=\frac{1-m}{2}\left(\frac{\pi^{2}}{a^{2}}+\frac{\pi^{2}}{b^{2}}+\frac{1+m}{2}\right)^{-1}
\end{gathered}
$$

by $(2.7),(2.14)$ and $(2.15)$. For any rectangle $\mathscr{R}_{\mathbb{B}}$ it is clear that, 


$$
0<\beta<1 .
$$

This means that the operator $K f$ in $(2.2)$ is a contraction mapping on the set $\varphi$ thereby ensuring the uniqueness of the solution $\varphi$ of (2.1).

To establish (2.10) for the operator $F$ defined by (2.1), we observe that

$$
\begin{gathered}
\left\|F \varphi_{1}-F \varphi_{2}\right\|=\left\|\varphi_{1}-\varphi_{2}-\left\{K f\left(\varphi_{1}\right)-K f\left(\varphi_{2}\right)\right\}\right\| \\
\geq\left\|\varphi_{1}-\varphi_{2}\right\|-\left\|K f\left(\varphi_{1}\right)-K f\left(\varphi_{2}\right)\right\| \\
\geq\left\|\varphi_{1}-\varphi_{2}\right\|-\beta\left\|\varphi_{1}-\varphi_{2}\right\| \text { by }(2.9) \\
=\gamma\left\|\varphi_{1}-\varphi_{2}\right\|
\end{gathered}
$$

where

$$
\gamma=1-\beta
$$

and $\gamma>0$ by (2.17). This proves (2.10).

Finally, we note that if the parameter $\sigma$ is not introduced in (1.14) and (1.15), the analysis leads to the parameter values

$$
\alpha=1, \quad \beta=\frac{a^{2} b^{2}}{\pi^{2}\left(a^{2}+b^{2}\right)}, \quad \gamma=1-\beta,
$$

and since for $K f$ to be a contraction we require $0<\beta<1$, it follows that the values of $a$ and $b$ that can be considered must not be too large. Since we wish to avoid any restriction on the size of the rectangle, we have developed the formulation based on (1.15).

\section{Pointwise Bounds for the Solution}

By the formulation of Section 2, the function $\varphi(x, y)$ satisfies the integral equation

$$
\begin{gathered}
\varphi(x, y)=\int_{0}^{b} \int_{0}^{a} k(x, y ; s, t) f(\varphi(s, t)) d s d t \\
=\langle k, f(\varphi)\rangle,
\end{gathered}
$$

where the kernel $k$ and the function $f$ are given in (2.3) and (1.15), and the inner product is that defined in (1.17) with integration over the variables $s$ and $t$ here.

Consider the difference

$$
\Delta=\langle k, f(\varphi)\rangle-\langle k, f(\Phi)\rangle
$$

where $\Phi$ is any function in $\varphi$. Then

$$
\begin{gathered}
|\Delta|=|\langle k, f(\varphi)-f(\Phi)\rangle| \\
\leq\|k\|\|f(\varphi)-f(\Phi)\|
\end{gathered}
$$




$$
\begin{aligned}
& \leq\|k\| \alpha\|\varphi-\Phi\| \text { by }(2.8) \\
\leq & \|k\| \frac{\alpha}{\gamma}\|F \varphi-F \Phi\| \quad \text { by }(2.10) \\
= & \|k\| \frac{\alpha}{\gamma}\|F \Phi\|=C(\Phi) \text { say }
\end{aligned}
$$

where we have used the fact that $F \varphi=0$. This result provides upper and lower bounds for $\langle k, f(\varphi)\rangle$, that is, $\varphi(x, y)$, which are

$$
\langle k, f(\Phi)\rangle-C(\Phi) \leq \varphi(x, y) \leq\langle k, f(\Phi)\rangle+C(\Phi) .
$$

These are the simple first order pointwise bounds for the solution $\varphi(x, y)$ of (1.1) with (1.3) which provide the basis of our calculations. They are subject to the conditions

$$
\left.\begin{array}{l}
\text { (i) } \Phi \text { belongs to } \varphi, \\
\text { (ii) }(2.8),(2.9) \text { and }(2.10) \text { hold }
\end{array}\right\} \text {. }
$$

More elaborate second order bounds for integral equations have been derived by Robinson and Yuen [8] but the bounds in (3.4) are sufficient for our purposes.

\section{Calculations}

To enable comparisons to be made with results obtained in previous work $[1,3]$, we shall include in our calculations two particular cases corresponding to $(i) a=1, b=1$, and $(i i) \quad a=\frac{1}{2}$, $b=\frac{1}{4}$.

We start the calculation by choosing the simple trial function

$$
\Phi=c u(x) v(y), u(x)=x(a-x), v(y)=y(b-y) .
$$

The form of this trial function has been chosen so that it satisfies the boundary condition (1.3). In order to calculate the bounds in (3.4), we require the value of

$$
K f(\Phi)=\int_{0}^{b} \int_{0}^{a} k(x, y ; s, t)\left\{e^{-\Phi(s, t)}+\sigma^{2} \Phi(s, t)\right\} d s d t .
$$

This involves evaluating integrals such as

$$
\int_{0}^{b} \int_{0}^{x} \sinh \mu_{n} s \sin \frac{n \pi t}{b} e^{-\Phi(s, t)} d s d t
$$

which cannot be calculated analytically and yet numerical integration is not suitable because of the oscillating function $\sin \frac{n \pi t}{b}$. To overcome this problem, we have used the series expansion

$$
e^{-\Phi}=e^{-c u v}=1-c u v+\frac{c^{2}}{2 !} u^{2} v^{2}-\frac{c^{3}}{3 !} u^{3} v^{3}+\ldots
$$

so that 


$$
K e^{-\Phi}=K 1-c K u v+\frac{c^{2}}{2 !} K u^{2} v^{2}-\frac{c^{3}}{3 !} K u^{3} v^{3}+\ldots
$$

and it is possible to integrate $K u^{m} v^{m}$ analytically. It is found that these functions have the form

$$
K u^{m} v^{m}=\sum_{n=1,3,5, \ldots}^{\infty} \sin \frac{n \pi y}{b} f_{m n}(x) .
$$

At each point $(x, y)$ of $\Re$ the pointwise bounds in (3.4) will be functions of $x$ and $y$ and of the parameter $c$ appearing in the trial function (4.1).

For each rectangle, $\Re$, we minimize the norm $\|F \Phi\|$ with respect to the parameter $c$, subject to the constraint $0<c \leq \frac{16 \bar{\varphi}}{a^{2} b^{2}}$ in order to ensure that $\Phi \in \varphi$. Here $\bar{\varphi}$ is a known global upper bound for the solution function $\varphi$. Initially we can take

$$
\bar{\varphi}=\max \psi(x, y) \text { over } \Re,
$$

where $\psi$ is given by (1.12). For instance, $\bar{\varphi}=0.007117$ when $a=\frac{1}{2}, b=\frac{1}{4}$, and $\bar{\varphi}=0.073671$ when $a=1, b=1$. The parameters $m, \sigma^{2}$ and $\gamma$, that are required in the bounds (3.4), are given by (2.12), (2.14) and (2.15).

These initial calculations based on (3.4) provide pointwise bounds for the solution function $\varphi(x, y)$ for a given rectangle, . Tables 1 and 2 display the results in the two cases $(i) a=\frac{1}{2}$, $b=\frac{1}{4}$ and $(i i) \quad a=1, b=1$.

Table 1: Initial bounds with $\Phi=c u v, a=\frac{1}{2}, b=\frac{1}{4}$

\begin{tabular}{|l|lll|}
\hline$y=0.1875$ & 0.00460176 & 0.00533950 & 0.00460176 \\
& 0.00460172 & 0.00533947 & 0.00460172 \\
$y=0.1250$ & 0.00603965 & 0.00707777 & 0.00603965 \\
& 0.00603961 & 0.00707773 & 0.00603961 \\
& & & \\
$y=0.0625$ & 0.00460176 & 0.00533950 & 0.00460176 \\
& 0.00460172 & 0.00533947 & 0.00460172 \\
\hline & $x=0.1250$ & $x=0.2500$ & $x=0.3750$ \\
\hline
\end{tabular}

Table 2: Initial bounds with $\Phi=c u v, a=1, b=1$

\begin{tabular}{|l|lll|}
\hline$y=0.75$ & 0.04330879 & 0.05461602 & 0.04330879 \\
& 0.04329879 & 0.05560435 & 0.04329879 \\
$y=0.5$ & & & \\
& 0.05461602 & 0.06992153 & 0.05461602 \\
& 0.05460435 & 0.06990746 & 0.05460435 \\
$y=0.25$ & & & \\
& 0.04330879 & 0.05461602 & 0.04330879 \\
& 0.04329879 & 0.05460435 & 0.04329879 \\
\hline & $x=0.25$ & $x=0.5$ & $x=0.75$ \\
\hline
\end{tabular}


For each rectangle considered, these calculations provide a new global upper bound for $\varphi(x, y)$ which we may call $\bar{\varphi}_{2}$. The corresponding parameters $m, \sigma^{2}$ and $\gamma$ are

$$
m_{2}=\exp \left(-\bar{\varphi}_{2}\right), \quad \sigma_{2}^{2}=\frac{1+m_{2}}{2}, \gamma_{2}=\frac{1-m_{2}}{2} .
$$

A second set of calculations can then be carried out using these parameter values. The process is then repeated until the bounds do not improve significantly.

To improve the bounds still further, we can take a new trial function

$$
\Phi=c_{1} u v+c_{2} u^{2} v^{2}
$$

involving two independent parameters, $c_{1}$ and $c_{2}$, where $u$ and $v$ are the functions defined in (4.1). Using the method of Hooke and Jeeves [6] and the procedure described above, we have repeated our calculations and found the optimum values of the parameters $c_{1}$ and $c_{2}$ by minimizing the norm $\|F \Phi\|$ subject to the condition that $\Phi \in \varphi$. Tables 3 through 6 contain the resulting pointwise bounds for $\varphi(x, y)$ for the illustrative cases, $(i) a=\frac{1}{2}, b=\frac{1}{4}, \quad(i i) \quad a=1$, $b=1,($ iii) $a=3, b=3$, (iv) $a=5, b=5$.

Table 3: Bounds with $\Phi=c_{1} u v+c_{2} u^{2} v^{2}, a=\frac{1}{2}, b=\frac{1}{4}$

\begin{tabular}{|l|lll|}
\hline$y=0.1875$ & 0.00460175 & 0.00533950 & 0.00460175 \\
& 0.00460173 & 0.00533947 & 0.00460173 \\
$y=0.1250$ & & & \\
& 0.00603965 & 0.00707777 & 0.00603965 \\
& 0.00603962 & 0.00707774 & 0.00603962 \\
$y=0.0625$ & & & \\
& 0.00460175 & 0.00533950 & 0.00460175 \\
& 0.00460173 & 0.00533947 & 0.00460173 \\
\hline & $x=0.1250$ & $x=0.2500$ & $x=0.3750$ \\
\hline
\end{tabular}

Table 4: Bounds with $\Phi=c_{1} u v+c_{2} u^{2} v^{2}, a=1, b=1$

\begin{tabular}{|l|lll|}
\hline$y=0.75$ & 0.04330437 & 0.05461043 & 0.04330437 \\
& 0.04330107 & 0.05460658 & 0.04330107 \\
$y=0.5$ & & & \\
& 0.05461043 & 0.06991351 & 0.05461043 \\
& 0.05460658 & 0.06990887 & 0.05460658 \\
$y=0.25$ & & & \\
& 0.04330437 & 0.05461043 & 0.04330437 \\
& 0.04330107 & 0.05460658 & 0.04330107 \\
\hline & $x=0.25$ & $x=0.5$ & $x=0.75$ \\
\hline
\end{tabular}


Table 5: Bounds with $\Phi=c_{1} u v+c_{2} u^{2} v^{2}, a=3, b=3$

\begin{tabular}{|l|lll|}
\hline $\mathrm{y}=2.25$ & 0.30076727 & 0.37064776 & 0.30076727 \\
& 0.29938460 & 0.36909138 & 0.29938460 \\
$\mathrm{y}=1.5$ & & & \\
& 0.37064776 & 0.46478145 & 0.37064776 \\
& 0.36909138 & 0.46397918 & 0.36909138 \\
$\mathrm{y}=0.75$ & & & \\
& 0.30076727 & 0.37064776 & 0.30076727 \\
& 0.29938460 & 0.36909138 & 0.29938460 \\
\hline & $\mathrm{x}=0.75$ & $\mathrm{x}=1.5$ & $\mathrm{x}=2.25$ \\
\hline
\end{tabular}

Table 6: Bounds with $\Phi=c_{1} u v+c_{2} u^{2} v^{2}, a=5, b=5$

\begin{tabular}{|l|lll|}
\hline$y=3.75$ & 0.58136541 & 0.67411656 & 0.58136541 \\
& 0.56427161 & 0.65962746 & 0.56427161 \\
$y=2.5$ & & & \\
& 0.67411656 & 0.80953283 & 0.67411656 \\
& 0.65962746 & 0.79912514 & 0.65962746 \\
$y=1.25$ & & & \\
& 0.58136541 & 0.67411656 & 0.58136541 \\
& 0.56427161 & 0.65962746 & 0.56427161 \\
\hline & $x=1.25$ & $x=2.5$ & $x=3.75$ \\
\hline
\end{tabular}

From Tables 1, 2, 3 and 4, it can be seen, as expected, that the bounds obtained by using (4.8) are closer together than the bounds obtained by using (4.1).

If we pick out the estimates for $\varphi(x, y)$ at the midpoint, we find that

$$
\left.\begin{array}{ll}
a=\frac{1}{2}, b=\frac{1}{4}: & 0.00707774 \leq \varphi\left(\frac{1}{4}, \frac{1}{8}\right) \leq 0.00707777, \\
a=1, b=1: & 0.06990887 \leq \varphi\left(\frac{1}{2}, \frac{1}{2}\right) \leq 0.06991351 .
\end{array}\right\}
$$

For comparison, we note that in the case $a=\frac{1}{2}, b=\frac{1}{4}$, Bellman and Kalaba [3] obtained the approximate value

$$
\varphi\left(\frac{1}{4}, \frac{1}{8}\right)=0.007071
$$

which is slightly too small according to our results. Also the earlier variation calculations [1] gave

$$
\begin{gathered}
a=\frac{1}{2}, b=\frac{1}{4}: \quad \varphi\left(\frac{1}{4}, \frac{1}{8}\right)=0.00769 \\
a=1, b=1: \quad \varphi\left(\frac{1}{2}, \frac{1}{2}\right)=0.1061
\end{gathered}
$$

which both lie considerably above the upper bound results in (4.9). 


\section{Concluding Remarks}

We have shown that, for the test cases considered, the first order pointwise bounds (3.4) are very effective in providing good estimates of the solution of the boundary value problem in (1.1) and (1.3). Much better results would be expected from the more elaborate second order bounds, involving two independent trial functions $\Phi$ and $\Psi$, given in equations (3.15) to (3.17) of [8], but calculations based on these, with the form of $\Phi$ as in (4.8) and the second function $\Psi$ chosen to be $\Psi=d$ and $\Psi=d u v$ where $d$ is a parameter that is optimized, lead only to a slight improvement on the results presented here.

\section{Acknowledgements}

We are indebted to the UK Science and Engineering Research Council for the award of a Research Studentship to Janet Clegg, and to the Commonwealth Scholarship Commission in the UK for the award of a Scholarship to Atulya Nagar.

\section{References}

[1] Anderson, N. and Arthurs, A.M., Variational solutions of the Liouville equation, Nuovo Cimento 56B (1968), 198-200.

[2] Bateman, H., Partial Differential Equations, The MacMillan Co., New York 1959.

[3] Bellman, R.E. and Kalaba, R.E., Quasilinearization and Nonlinear Boundary-Value Problems, Elsevier Publishing Co., New York 1965.

[4] Buck, R.C., Advanced Calculus, McGraw-Hill, New York 1956.

[5] Friedman, B., Principles and Techniques of Applied Mathematics, Wiley, New York 1956.

[6] Hooke, R., and Jeeves, T.A., Direct search solution of numerical and statistical problems, J. Assn. Comput. Mach. 8 (1961), 212-229.

[7] Protter, M.H. and Weinberger, H.F., Maximum Principles in Differential Equations, Prentice-Hall, Englewood Cliffs, New Jersey 1967.

[8] Robinson, P.D. and Yuen, P.K, Bivariational methods for Hammerstein integral equations, IMA J. App. Math. 39 (1987), 177-188.

[9] Stakgold, I., Boundary Value Problems of Mathematical Physics, Vol. 1, The MacMillan Co., New York 1967. 


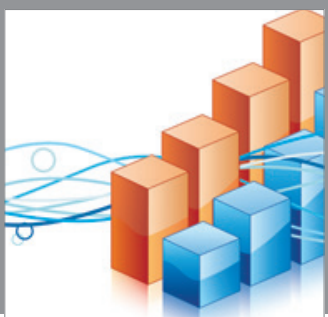

Advances in

Operations Research

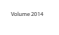

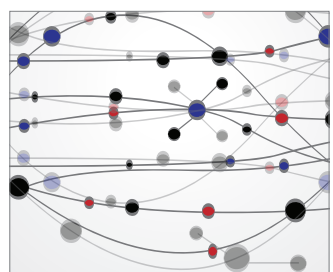

\section{The Scientific} World Journal
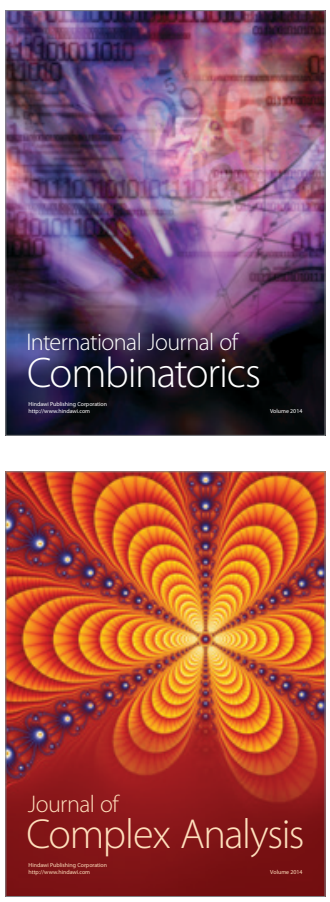

International Journal of

Mathematics and

Mathematical

Sciences
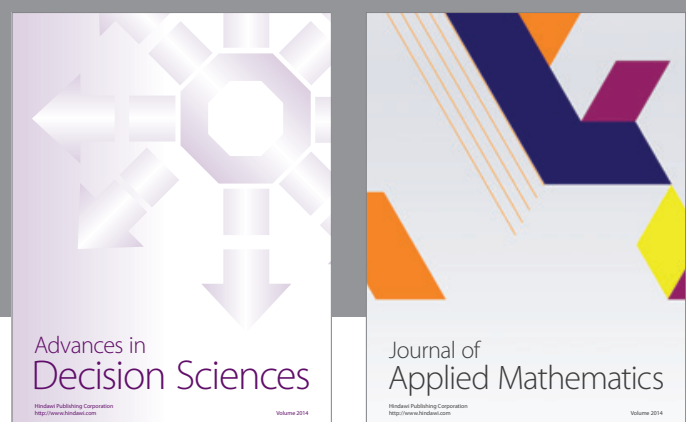

Journal of

Applied Mathematics
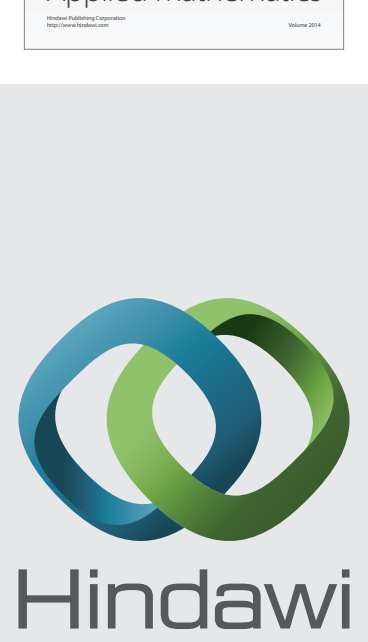

Submit your manuscripts at http://www.hindawi.com
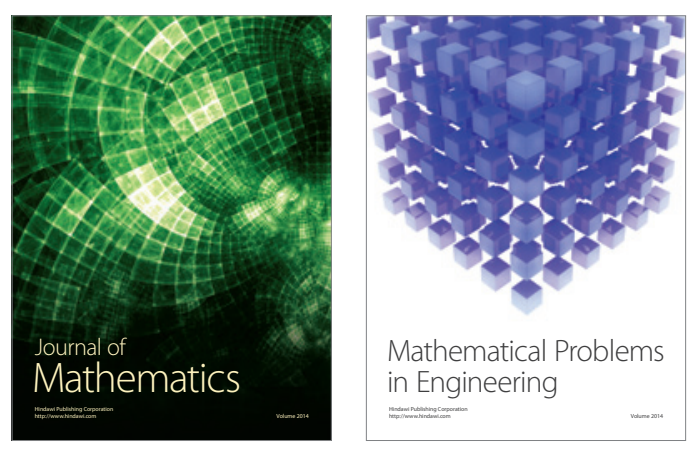

Mathematical Problems in Engineering
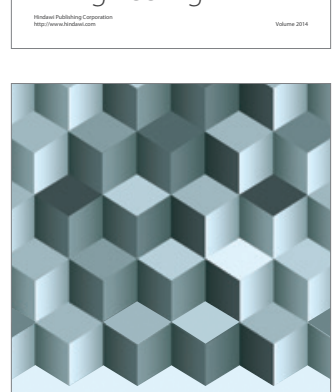

Journal of

Function Spaces
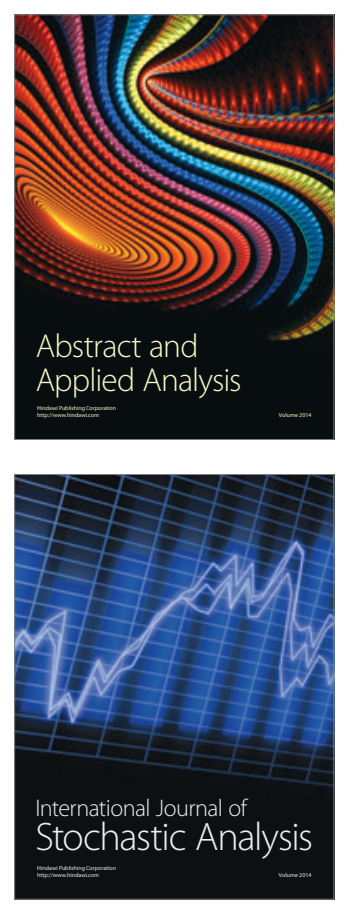

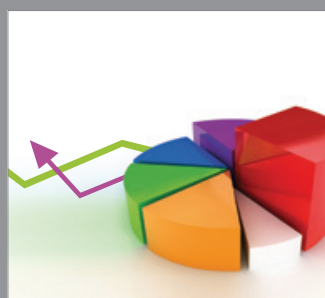

ournal of

Probability and Statistics

Promensencen
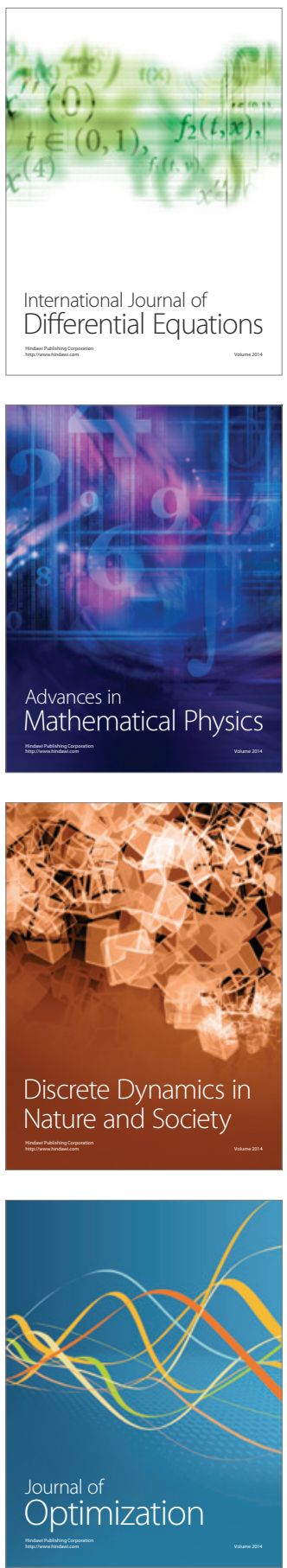